\title{
Stochastic modelling of wind and its implication for wildfire spread predictions
}

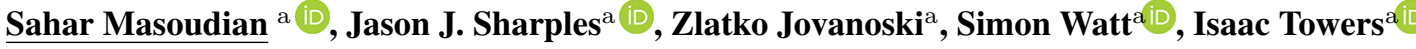 \\ ${ }^{a}$ School of Science, UNSW Canberra, Australia \\ Email: s.masoudian@adfa.edu.au
}

\begin{abstract}
Wildfire events have received much attention recently due to their serious socioeconomic impacts in countries like Australia, Canada, the United States and Greece. To tackle wildfire crises, authorities and decision makers rely on a capability to obtain accurate and informative predictions of wildfire spread to support real time decision making, often with life or death consequences.

However, the uncertainty inherent in wildfire model inputs, such as wind speed and direction, makes accurate prediction of wildfire spread a challenging $t$ ask. To a ccommodate the inherent uncertainties of the wildfire environment, wildfire modellers a re increasingly drawing u pon probabilistic or ensemble-based approaches to modelling fire s pread. For instance, Cruz [2010] used a Gaussian probability distribution function (PDF), with ensemble members sampled using a Monte Carlo approach, to simulate the variation of wind speed over a range of 27-78 $\mathrm{km} \mathrm{h}^{-1}$. In such simulations, spread prediction relies on different probable scenarios rather than a single set of input values. For example, the FireDST framework [French et al., 2014] samples input weather conditions from a uniform distribution, then visualizes the simulator output using burn probability maps, which provide useful information on the likelihood of different parts of the landscape being impacted by fire over a specific period of time [Pinto et al., 2016].

Although ensemble-based approaches can potentially capture the uncertainty in the process of fire modelling, all of the approaches currently in use rely on deterministic simulators to derive burn probability maps. Deterministic simulators provide a single output from a given set of input conditions, and so do not faithfully represent the inherent stochasticity of real fire p ropagation. In this study, we consider an alternate modelling approach that better acknowledges the intrinsic uncertainties associated with wildfire spread.

Wind speed and direction are critical inputs for bushfire simulation as a significant portion of the uncertainty in the wildfire modelling is caused by spatial and temporal variations in the wind. To capture this uncertainty, one can treat wind speed and direction as stochastic variables; this permits more faithful incorporation of the stochasticity of input variables into the simulation process, and provides a way of obtaining burn probability maps that better reflect the inherent uncertainties of wildfire prediction.
\end{abstract}

A variety of stochastic processes can be used to model wind speed and direction. While it is still not known which process is the best to use in the context of wildfire simulation, we focused on two that have previously appeared in the wind and fire modelling 1 iterature. The Wiener process has used by Zazali et al. [2017] to model fire s pread, w hile the First-Order G auss-Markov p rocess ( also c alled the O rnstein-Uhlenbeck (OU) process) has received a lot of attention in wind forecasting and for many other environmental factors [Edwards and Hurst, 2001].

In this study, the Wiener and First-Order Gauss-Markov (FOGM) process models are employed to model wind speed and wind direction. The process noise $\delta$ of each process is calibrated using a series of data collected from eleven Davis Vantage Pro2 automatic weather stations that were set up at an experimental field site [Quill, 2017]. The wind vector is then simulated by the two stochastic processes using the estimated values of the process noise for each of the two processes. In the final step, we evaluated the models by calculating the root mean square error (RMSE) and the standard deviation of the error (SDE), and compare the distributions of simulated data by stochastic models and the observed data.

Finally, the stochastic wind models were incorporated within the SPARK fire simulation framework [Hilton et al., 2015], to produce representations of fire s pread based o $\mathrm{n}$ s tochastic w ind fi elds wi th the calibrated process noise levels. The outcomes of the stochastic fire spread simulations provided a different interpretation of fire spread compared to simulations based on deterministic ensembles.

Keywords: Stochastic modelling, wildfire spread, wind, Wiener Process, First Order Gauss-Markov Process 


\section{INTRODUCTION}

Wildfire simulations provide spatiotemporal information on the likely propagation of a wildfire across the landscape, which can be used to aid in critical decision making. However, there is still much uncertainty associated with these simulations. Model uncertainty can arise for a variety of reasons; for example, lack of model applicability, internal model inaccuracy, and input data errors [Cruz, 2010]. There are many inputs that can cause scientific uncertainty in fire propagation models, including topography, weather, and fuel conditions. Specifically, high variability of meteorological inputs can be a significant source of uncertainty in wildfire models. Perhaps the most important meteorological factor in fire prediction is the wind; but it is also the variable associated with the highest degree of uncertainty |Quill et al. 2019]. As a consequence, there is potential to improve fire spread modelling by better capturing uncertainty in wind fields using more sophisticated statistical approaches.

One such approach is to use a deterministic ensemble method. Wind inputs are sampled to form a set of variable input ensemble members, each of which are considered as constant over the course of a simulation. A deterministic fire simulator is then used to derive a range of fire spread outputs, each corresponding to a single ensemble wind input. An alternative approach is to treat the wind as a realisation of a stochastic process, so that at each time step, the simulation incorporates previous variations in wind direction and magnitude. This means that at each time step within a simulation, the wind varies in a way that depends on the state of the wind in the previous step. In this paper, the step size in Spark fire simulations are considered 10 seconds.

Zazali et al. [2017] modified a basic deterministic wildfire propagation model by incorporating a Wiener process model to describe stochasticity in wind speed and direction. They compared a deterministic ensemble approach with the stochastic model, and found that the stochastic model produced a broader range of probable fire propagation scenarios than the ensemble approach.

In addition to fire modelling considerations, much attention has been given to modelling wind in order to forecast wind speed or its power in a stochastic way. Bivona et al. [2011| developed and then evaluated a stochastic model called Box-Jenkins for hourly averaged wind speed time series for two regions in Italy over 4 years. Wind data (magnitude and direction) at altitudes of up to $20 \mathrm{~km}$ were used by Turkoglu [2014] to stochastically model wind via an Ornstein-Uhlenbeck stochastic process. The Ornstein-Uhlenbeck process is the same as the First Order Gauss-Markov (FOGM) process used by Benth et al. [2018].

Indeed, the FOGM stochastic process has been frequently utilised for wind modelling in power systems. It has been used to:

1. simulate the fluctuation of wind speed to generate a power law distribution. [Edwards and Hurst, 2001];

2. model the dynamic behaviour of wind power for wind storage applications in power systems [Hernández Ávalos et al., 2015];

3. generate wind speed trajectories with statistical properties similar to those observed in an historical wind speed sample [Zárate-Miñano et al. , 2013];

4. model wind speed via an Ornstein-Uhlenbeck Geometric Brownian Motion model, while including its long-term daily cycle [Loukatou et al., 2018].

In this study, two stochastic processes Wiener and FOGM processes are employed to model empirical wind data. Then, these calibrated stochastic wind models are implemented in a fire spread simulator called Spark to get a better representation of the intrinsic variability of fire spread.

\section{METHODS AND DATA}

The Wiener and FOGM processes can be defined by the difference equations (1) and (2), respectively;

$$
\begin{aligned}
& x_{k+1}=x_{k}+\delta^{\prime} \sqrt{d t} N(0,1), \\
& x_{k+1}=\Phi_{k} x_{k}+\delta N(0,1)
\end{aligned}
$$

where $x_{k}$ is wind speed or direction at time $k$, the transition function $\Phi_{k}=\exp (-d t / \tau), d t$ is the time difference between the two states $x_{k+1}$ and $x_{k}$, and the FOGM process noise $\delta$ is given by

$$
\delta=\sigma \sqrt{1-\exp \left(-\frac{2 d t}{\tau}\right)} .
$$


In equation (3), $\sigma$ is the standard deviation of the data, and $\tau$ is the correlation time of the process. This factor is the time within which the data points are assumed correlated with each other. After trying different correlation times $(1,5,10,24$ hours), a correlation time of 5 hours for wind speed and 1 hour for wind direction were found to be reasonable for all the cases considered in this study.

An important factor in the stochastic processes described above are the values for the process noise $\left(\delta^{\prime}\right.$ and $\left.\delta\right)$, which can be estimated from wind observations. In different studies, the process noise is estimated differently, depending on the data used. Zazali et al. [2017], took the Wiener process noise as $\delta^{\prime}=5$ for the wind speed $\delta^{\prime}=\pi / 18$ for the wind direction. In their work on modelling wind for energy markets, Benth et al. [2018], estimated the FOGM process noise as $\delta \cong 0.339$ with a $95 \%$ confidence interval of $[0.327,0.352]$.

The process noises required to model wind speed and direction were estimated using wind data recorded by eleven Davis 1 Vantage Pro $2^{\mathrm{TM}}$ Portable Automatic Weather Stations (labelled P1-P11) Quill |2017|. These data were collected over the 57 day period 14 February - 11 April, 2014 (except at P9, whose data only covers the period 14-27 February, 2014). Data were collected at an open field site near Canberra airport (Australian Capital Territory, Australia) [Quill, 2017|. We considered wind speed ( $\mathrm{m} \mathrm{s}^{-1}$ ) and wind direction (16 compass points converted to degrees) data for all 57 days with a time interval of 5 minutes.

By considering hourly variations of both wind speed and direction, calculating the point to point variance, and applying equations (1) and (3), the process models for wind speed and direction can be calibrated by estimating the values of the process noise for both Wiener and FOGM stochastic processes. This calibration was performed for each station P1-P11, with the process noise estimated over hourly blocks (the duration of blocks was selected based on the error of the simulation). Figures (1) and (2) show the frequency of the different estimated values for process noises of wind speed for every hour for station $P 1$.

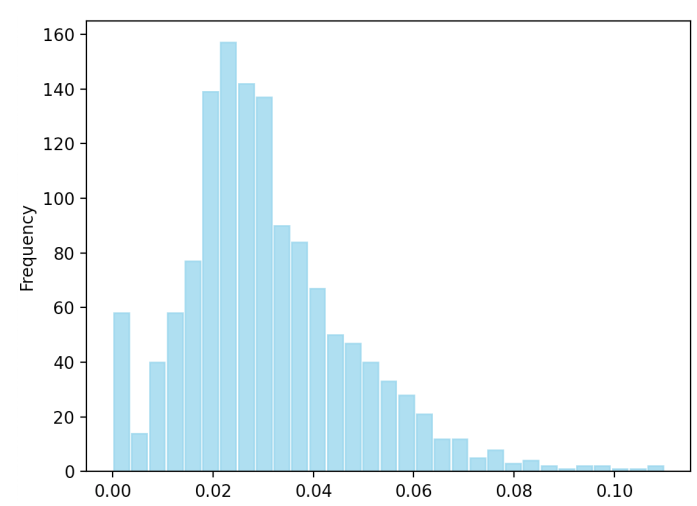

Figure 1. Histogram plot on estimated values for Wiener process noise $\delta^{\prime}$ for wind speed for every hour for station P1.

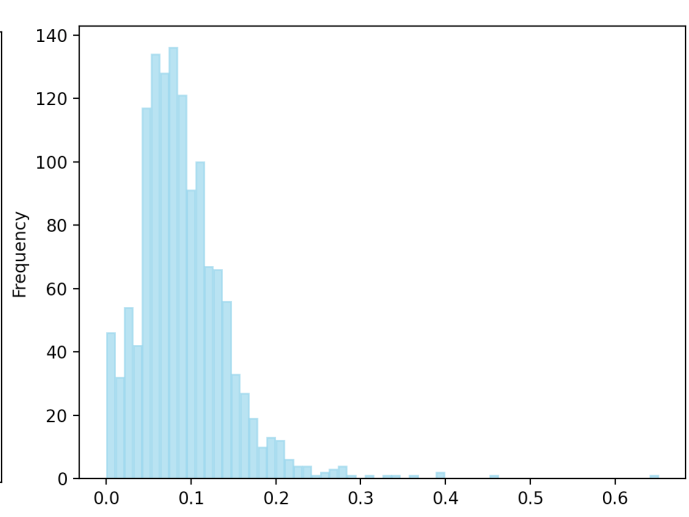

Figure 2. Histogram plot on estimated values for FOGM process noise $\delta$ for wind speed for every hour for station P1.

\section{RESULTS AND DISCUSSION}

The Wiener and FOGM process noises were estimated for both wind speed and direction at all weather stations. The process noises were estimated for each one hour interval at each weather station. The medians of all these values (for wind speed and direction) were then used as the values of the process noises in (1) and (2). For fire simulations, the median of all the estimated values for each station is implemented, these values are $\left\{\delta^{\prime}=0.026, \delta=0.084\right\}$ for wind speed, and $\left\{\delta^{\prime}=0.894, \delta=5.996\right\}$ for wind direction.

In order to compare the data predicted by the two stochastic models (Wiener and FOGM) with the estimated process noises to the observed data, the Root Mean Square Error (RMSE) between the simulated and observed data is calculated.

If $\hat{w}_{i}$ are the predicted data using a stochastic process with standard deviation $\hat{\sigma}$ for the duration (50 days with step size of 5 minutes) there is data available, and $w_{i}$ are the observed data with the standard deviation $\sigma$, then 
the RMSE is calculated as,

$$
\mathrm{RMSE}=\sqrt{\sum_{i=1}^{n} \frac{\left(\hat{w}_{i}-w_{i}\right)^{2}}{n}}
$$

where $n$ is the number of data points.

\subsection{RESULTS ON WIND SPEED AND DIRECTION}

The median value of all values of the estimated Wiener process noises, FOGM process noise, and their RMSE for wind speed were taken as the vales defining the stochastic processes.

Because of the stochastic nature of these models, each time the simulations are run, they give slightly different results, with different resultant RMSE. Thus for any evaluation, 100 Monte-Carlo simulations were performed, then the median of all these 100 members were considered as the calculated errors. The calculated RMSE values in Table (1) show that there are only small differences between the observed data and predicted data generated by the Wiener and FOGM processes for both wind speed and direction. The maximum RMSE between the observed and simulated wind speed data was approximately $3 \mathrm{~km} \mathrm{~h}^{-1}$ for the Wiener, and $2 \mathrm{~km}$ $\mathrm{h}^{-1}$ for the FOGM process. Also, in Table (2) the RMSE for wind direction has maximum values of about $40^{\circ}$ for both the Wiener and the FOGM processes.

\begin{tabular}{|c|c|c|c|c|}
\hline Station & Median of $\delta^{\prime}$ Wiener & Median of RMSE Wiener & Median of $\delta$ FOGM & Median of RMSE FOGM \\
\hline P1 & 0.027 & 0.9818 & 0.084 & 0.438 \\
\hline P2 & 0.027 & 0.944 & 0.085 & 0.537 \\
\hline P3 & 0.026 & 0.920 & 0.082 & 0.547 \\
\hline P4 & 0.027 & 0.957 & 0.086 & 0.563 \\
\hline P5 & 0.026 & 0.906 & 0.084 & 0.527 \\
\hline P6 & 0.026 & 0.966 & 0.081 & 0.565 \\
\hline P7 & 0.026 & 0.937 & 0.082 & 0.582 \\
\hline P8 & 0.025 & 0.792 & 0.077 & 0.432 \\
\hline P9 & 0.033 & 1 & 0.104 & 0.616 \\
\hline P10 & 0.026 & 0.858 & 0.081 & 0.470 \\
\hline P12 & 0.025 & 0.938 & 0.079 & 0.572 \\
\hline
\end{tabular}

Table 1. Estimated process noise $\delta^{\prime}$ for the Wiener and $\delta$ for the FOGM process models from the calibration of wind speed time series, and the median values of the Root Mean Square Error (RMSE) between the simulated data using the estimated process noises and the observed data for all 11 weather stations. Every estimation has been performed for each hour, and units are $\mathrm{m} \mathrm{s}^{-1}$.
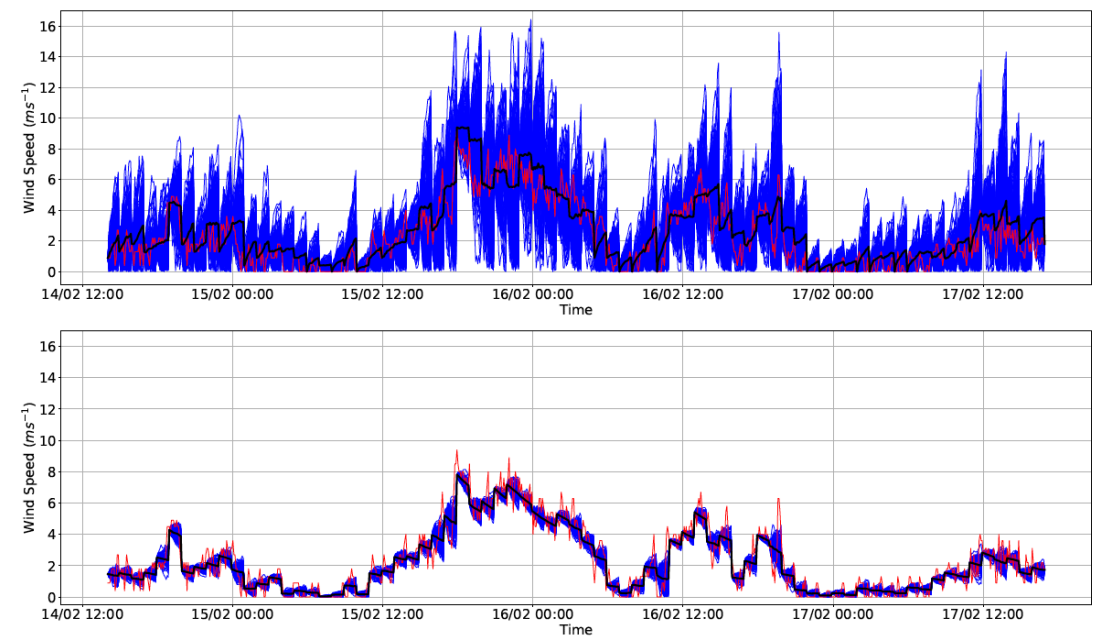

Figure 3. Wind speed time series of 100 Monte-Carlo simulations (blue line) using the Weiner process with the estimated $\delta^{\prime}=0.026$ (upper), and the First-Order-Gauss-Markov (FOGM) with estimated $\delta=0.084$ (lower); the mean of all these simulations (black line), and the observed values (red line) for the duration of 3 days for station $P 1$. 
The wind speed and direction time series in Figure (3) and (4) represent the 100 Monte-Carlo Wiener and FOGM simulations using the estimated process noises, for $14^{\text {th }}-16^{\text {th }}$ February, as well as the observed data for this period. It can be seen that the observed values fall well within the simulated lower and upper bounds, with the mean simulation resembling the observed values. While the Wiener process exhibits a wider range of uncertainty, which may be helpful in managing the uncertainty in fire models, the FOGM process typically remains close to the observed data, which gives very similar simulations to the observed values at all the ensemble members.

\begin{tabular}{|c|c|c|c|c|}
\hline Station & Median of $\delta^{\prime}$ Wiener & Median of RMSE Wiener & Median of $\delta$ FOGM & Median of RMSE FOGM \\
\hline P1 & 0.809 & 42.201 & 5.442 & 44.423 \\
\hline P2 & 0.794 & 45.229 & 5.393 & 45.103 \\
\hline P3 & 0.867 & 41.923 & 5.414 & 43.366 \\
\hline P4 & 0.668 & 33.642 & 4.346 & 41.786 \\
\hline P5 & 0.885 & 46.878 & 5.642 & 45.078 \\
\hline P6 & 0.783 & 39.898 & 5.246 & 44.574 \\
\hline P7 & 0.867 & 45.838 & 6.311 & 40.057 \\
\hline P8 & 0.929 & 48.495 & 6.267 & 43.499 \\
\hline P9 & 1.512 & 64.684 & 10.615 & 51.034 \\
\hline P10 & 0.946 & 51.506 & 6.362 & 45.838 \\
\hline P12 & 0.783 & 41.586 & 4.921 & 45.936 \\
\hline
\end{tabular}

Table 2. Estimated process noise $\delta^{\prime}$ for the Wiener and $\delta$ for the FOGM process models from the calibration of wind direction time series, and the median values of the Root Mean Square Error (RMSE) between the simulated data using the estimated process noises and the observed data for all 11 weather stations. Every estimation has been performed for each hour, and units are degrees.
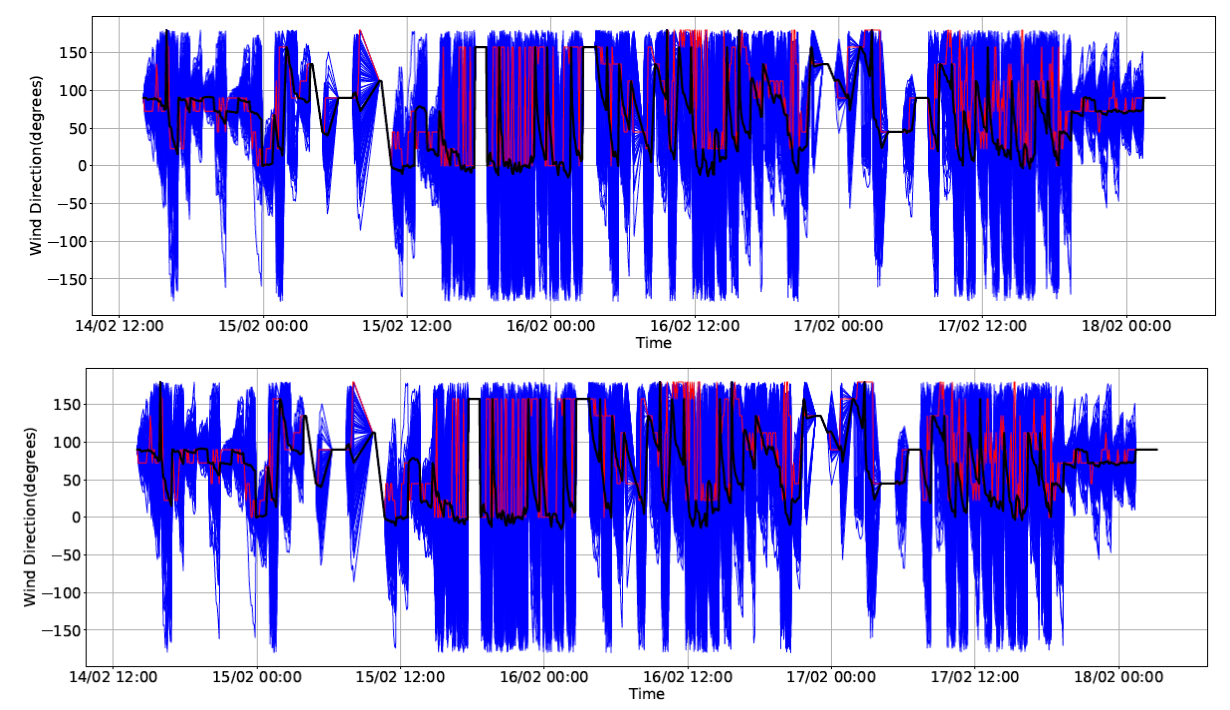

Figure 4. Wind direction time series of 100 Monte-Carlo (blue lines) using the Wiener process with estimated $\delta^{\prime}=0.894$ (upper), and the First-Order-Gauss-Markov (FOGM) with estimated $\delta=0.084$ (lower), the mean of all the simulations (black line), and the observed values (red line) for the duration of 3 days for station $P 1$.

\section{STOCHASTIC WILDFIRE SIMULATION IN THE SPARK FRAMEWORK}

The SPARK fire simulator is able to model the 2-D growth of a fire perimeter using the level set method for the tracking the interface [Miller et al., 2015]:

$$
\frac{\partial \phi}{\partial t}=-s|\Delta \phi|
$$

where $s$ is the normal speed of fire perimeter, and $\phi$ is the signed distance from the fire interface ( which is positive outside the fire interface, and negative inside the interface) [Sethian, 1999]. In this study, the 
fire perimeter is assumed to evolve at each point of the fire front as an ellipse, based on Huygens' principle [Anderson et al., 1982]. This elliptic fire propagation is described by |Roberts, 1993]:

$$
s=g \cdot \dot{n}+\sqrt{h^{2}+\left(f^{2}-h^{2}\right) \dot{n}^{2}} .
$$

where $\{f, g, h\}$ are the shape parameters, which determine the length and breadth of the elliptical fire as a function of the wind speed, and $\dot{n}=\hat{\mathbf{n}} \cdot \hat{\mathbf{w}}$ ( $\hat{\mathbf{n}}$ is the normal vector to the curve and $\hat{\mathbf{w}}$ is the unit wind vector). The wind vector $\mathbf{w}$ can be decomposed along standard axis directions as $w \cos \theta$ and $w \sin \theta$ where $w=\|\mathbf{w}\|$ is the wind speed.

To incorporate stochasticity in fire simulations within the SPARK framework, difference equations (1), (2), and the estimated values of $\delta^{\prime}, \delta$ based on observed data of both wind speed and direction are applied, such that $w$ and $\theta$ are updated in SPARK fire simulations over time. The stochastic simulations are then repeated to achieve 1000 realisations of the stochastic processes. To visualise these fire spread models, burn probability maps are shown in Figure (5). One iteration of a deterministic elliptical fire model is also shown in white.

It is still debatable which of the two approaches, the 1000 realisations-stochastic fire model and the ensemble simulation of a single deterministic fire model ( Figure(5)), can model fire propagation more faithfully in terms of the expressing the existing uncertainty in this environmental event. However, allowing the wind to vary stochastically from one time step to the next, does seem to better represent the intrinsic fluctuations in wind. This will be an on-going area of investigation.
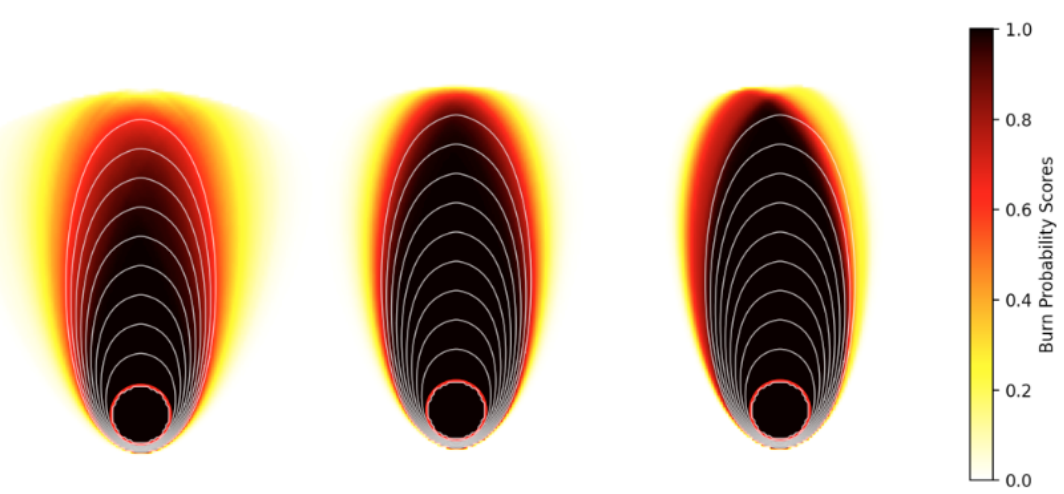

Figure 5. Burn probability map of SPARK simulation for fire growth during 100 seconds with a circle as an initial ignition by implementing different simulations: (left) Ensemble method with 1000 realisations where wind speed and direction were normally distributed with standard deviations $5 \mathrm{~ms}^{-1}, 10^{\circ}$, and arbitrary mean values; (middle) Wiener process for wind speed and wind direction with $\delta_{\text {Speed }}^{\prime}=0.026$ and $\delta_{\text {Direction }}^{\prime}=0.894$; (right) FOGM process for wind speed and wind direction with $\delta_{\text {Speed }}^{\prime}=0.084$ and $\delta_{\text {Direction }}^{\prime}=5.996$. In all fire simulations, the initial wind is southerly with magnitude $20 \mathrm{~ms}^{-1}$. The white contours show a single deterministic fire spread during 100 seconds when wind blows along y-axis with magnitude $20 \mathrm{~ms}^{-1}$, and the contour interval is 10 seconds.

\section{CONCLUSIONS}

In this paper two different stochastic processes Wiener process and First-Order-Gauss-Markov (FOGM), were used to account for the uncertainty in wildfire spread that arises due to the intrinsic temporal variation of wind speed and direction. Wind stochasticity was incorporated through calibration of the stochastic processes using wind data collected from a Canberra field site in 2014. Evaluation of these processes showed that the observed data mostly fell within the range of the stochastic models, and the mean of these models behaves as the observed values, but were slightly different for each process. By applying the estimated values of process noises, the stochastic wind field were implemented in the SPARK fire propagation framework. The resulting 'stochastic' fire simulations were presented as different probability maps as an alternative to deterministic ensemble simulations, which could be further investigated to study the reliability of fire propagation forecasts for each simulation. The analyses undertaken in this study to estimate the process noises were based on one case study at one field-site. More analyses on different sites with different topographic conditions can be performed to understand the influence of complex terrain and other environmental features (e.g. forest canopy) on estimates of process noise of stochastic wind fields. 


\section{REFERENCES}

Anderson, D., E. Catchpole, N. De Mestre, and T. Parkes (1982). Modelling the spread of grass fires. The ANZIAM Journal 23(4), 451-466.

Benth, F. E., L. Di Persio, and S. Lavagnini (2018). Stochastic modeling of wind derivatives in energy markets. Risks 6(2), 56.

Bivona, S., G. Bonanno, R. Burlon, D. Gurrera, and C. Leone (2011). Stochastic models for wind speed forecasting. Energy conversion and management 52(2), 1157-1165.

Cruz, M. G. (2010). Monte Carlo-based ensemble method for prediction of grassland fire spread. International Journal of Wildland Fire 19(4), 521-530.

Edwards, P. J. and R. B. Hurst (2001). Level-crossing statistics of the horizontal wind speed in the planetary surface boundary layer. Chaos: An Interdisciplinary Journal of Nonlinear Science 11(3), 611-618.

French, I. A., T. J. Duff, R. B. P. Cechet, K. G. Tolhurst, J. D. Kepert, and M. Meyer (2014, 2014). FireDST: a simulation system for short-term ensemble modelling of bushfire spread and exposure, pp. 1147-1158. Coimbra: Imprensa da Universidade de Coimbra.

Hernández Ávalos, J., P. Johnson, and P. Duck (2015). Sladi: a semi-lagrangian alternating-direction implicit method for the numerical solution of advection-diffusion problems with application to electricity storage valuations. Journal of Computational Finance 19(2), 69-108.

Hilton, J., L. Hetherton, C. Miller, A. Sullivan, and M. Prakash (2015). The SPARK framework. Technical report, Technical Report, Report Number: EP152898.

Loukatou, A., S. Howell, P. Johnson, and P. Duck (2018). Stochastic wind speed modelling for estimation of expected wind power output. Applied energy 228, 1328-1340.

Miller, C., J. Hilton, A. Sullivan, and M. Prakash (2015). SPARK-a bushfire spread prediction tool. In International Symposium on Environmental Software Systems, pp. 262-271. Springer.

Pinto, R. M., A. Benali, A. C. Sá, P. M. Fernandes, P. M. Soares, R. M. Cardoso, R. M. Trigo, and J. M. Pereira (2016). Probabilistic fire spread forecast as a management tool in an operational setting. SpringerPlus 5(1), 1205.

Quill, R. (2017). Statistical characterisation of wind fields over complex terrain with applications in bushfire modelling. Ph. D. thesis, School of Physical, Environmental and Mathematical Sciences, UNSW.

Quill, R., J. J. Sharples, N. S. Wagenbrenner, L. A. Sidhu, and J. M. Forthofer (2019). Modeling wind direction distributions using a diagnostic model in the context of probabilistic fire spread prediction. Frontiers in Mechanical Engineering 5, 5.

Roberts, S. (1993). A line element algorithm for curve flow problems in the plane. The ANZIAM Journal 35(2), 244-261.

Sethian, J. (1999). Level Set Methods and Fast Marching Methods: Evolving Interfaces in Computational Geometry, Fluid Mechanics, Computer Vision, and Materials Science. Cambridge Monographs on Applied and Computational Mathematics. Cambridge University Press.

Turkoglu, K. (2014, 02). Statistics based modeling of wind speed and wind direction in real time optimal guidance strategies via ornstein-uhlenbeck stochastic processes. In Fourth Aviation, Range, and Aerospace Meteorology Special Symposium, American Meteorological Society.

Zárate-Miñano, R., M. Anghel, and F. Milano (2013). Continuous wind speed models based on stochastic differential equations. Applied Energy 104, 42-49.

Zazali, H., I. Towers, and J. Sharples (2017, Jan). Incorporating environmental uncertainty in fire spread modelling. In G. Syme, D. Hatton MacDonald, B. Fulton, and J. Piantadosi (Eds.), Proceedings - 22nd International Congress on Modelling and Simulation, MODSIM 2017, pp. 1194-1200. https://www . mssanz.org.au/modsim2017/H10/zazali.pdf 